\title{
JACQUES THÉPOT
}

\section{Scrutin proportionnel et optimisation mathématique}

Revue française d'automatique, d'informatique et de recherche opérationnelle. Recherche opérationnelle, tome 20, $\mathrm{n}^{\circ} 2$ (1986), p. $123-138$.

<http://www.numdam.org/item?id=RO_1986_20_2_123_0>

C AFCET, 1986, tous droits réservés.

L'accès aux archives de la revue « Revue française d'automatique, d'informatique et de recherche opérationnelle. Recherche opérationnelle » implique l'accord avec les conditions générales d'utilisation (http://www.numdam.org/ legal.php). Toute utilisation commerciale ou impression systématique est constitutive d'une infraction pénale. Toute copie ou impression de ce fichier doit contenir la présente mention de copyright.

\section{Numdam}

Article numérisé dans le cadre du programme

Numérisation de documents anciens mathématiques

http://www.numdam.org/ 


\title{
SCRUTIN PROPORTIONNEL ET OPTIMISATION MATHÉMATIQUE $(*)$
}

\author{
par Jacques ThéPot $\left(^{\mathbf{1}}\right.$ )
}

\begin{abstract}
Résumé. - Cet article met en évidence les problèmes d'optimisation sous-jacents aux méthodes des plus forts restes et de la plus forte moyenne utilisées dans la répartition des sièges du scrutin proportionnel. On montre comment, dans les deux cas, il s'agit de minimiser une certaine fonction mesurant l'écart entre la distribution des suffrages et celle des sièges.
\end{abstract}

Mots clés : Programmation mathématique; choix collectif; vote.

Abstract. - This article shows how two methods commonly used to allocate the seats in a proportional voting system are based upon optimization problems. These methods can be interpreted as algorithms aimed at minimizing specific distance valuations between the distributions of the votes and the seats.

Keywords: Mathematical programming; collective choice; voting system.

\section{INTRODUCTION}

Au printemps 1985, le gouvernement déposa un projet de loi instaurant la représentation proportionnelle départementale (RP) pour l'élection des députés. Le débat politique suscité alors fut ce que nous savons (Portelli, [4]) : identique à ce qu'il est en France depuis que la république est la République, chaque fois que les gouvernements modifient les règles du jeu électoral: les arguments que l'on échange, les intentions que l'on prête et les arrières pensées que l'on nourrit alors de tous côtés font vraiment partie du patrimoine national.

Le problème de la répartition des restes est une pièce maîtresse de ce florilège. Le gouvernement a finalement opté pour la RP à la plus forte moyenne (PFM) dont l'expérience montre qu'elle accorde un certain avantage aux grosses listes au détriment des petites qui auraient été mieux loties si l'on avait choisi la RP aux plus forts restes (PFR).

(*) Reçu octobre 1985.

(1) Université Louis-Pasteur, Faculté des Sciences Économiques, et de Gestion, 4, rue BlaisePascal, 67070 Strasbourg Cedex.

R.A.I.R.O. Recherche opérationnelle/Operations Research, 0399-0559/86/01 12316/\$3.60

(C) AFCET-Gauthier-Villars. 
Ce que l'expérience nous enseigne, est-il possible de l'établir par le raisonnement? Nous nous proposons d'aborder ce problème en appliquant les principes de l'optimisation mathématique: nous montrerons dans cet article comment chacun de ces modes de scrutin s'interprète comme une procédure itérative de recherche de l'optimum d'une certaine fonction mesurant l'écart entre la distribution des sièges et celle des suffrages exprimés. Il apparaitra ainsi que la méthode PFR et la méthode PFM diffèrent fondamentalement dans l'évaluation de l'écart retenue. L'intérêt de cette approche est de fournir un cadre général et relativement simple qui puisse intégrer d'autres modes de scrutin et d'en saisir conceptuellement les particularités.

Cet article est divisé en quatre parties: après une première partie consacrée à la position du problème, nous étudierons, dans les deux suivantes respectivement la méthode PFR et la méthode PFM; les comparaisons et les commentaires feront l'objet de la quatrième partie.

\section{POSITION DU PROBLEME}

Considérons une circonscription électorale où $n$ sièges sont à pourvoir. Soit $q$ le nombre de listes en présence. La liste $i$ recueille $x_{i}$ suffrages sur un total de $S$ suffrages exprimés:

$$
\sum_{i=1}^{q} x_{i}=S
$$

Soit $p_{i}$ la proportion de voix obtenues par la liste $i$ :

$$
p_{i}=x_{i} / S
$$

d'où évidemment :

$$
\sum_{i=1}^{q} p_{i}=1
$$

Pour simplifier l'analyse, nous n'introduirons pas dans ce schéma de seuil minimum $(5 \%)$ en deçà duquel une liste n'a aucun élu. Cette hypothèse ne modifie pas la signification des résultats que nous obtiendrons.

Le problème consiste à déterminer les $n_{i}$ sièges affectés à la liste $i$ à partir de la proportion $p_{i}$ de voix obtenues. Si $n p_{i}$ est un entier, alors le nombre de sièges $n_{i}$ est égal à $n p_{i}$; c'est le principe de base du scrutin proportionnel. Toute la question est de savoir ce que l'on fait lorsque $n p_{i}$ n'est pas un entier; 
autrement dit, selon quel critère procède-t-on aux arrondis compte tenu de la contrainte:

$$
\sum_{i=1}^{q} n_{i}=n ?
$$

La méthode des plus forts restes et celle de la plus forte moyenne correspondent à deux matières différentes de réaliser ces arrondis. Illustrons cela sur un exemple.

\section{1. Illustration par un exemple numérique}

Nous considérons la situation suivante:

$$
\left\{\begin{array}{l}
\text { suffrages exprimés: } S=1000, \\
\text { nombre de listes: } q=3, \\
\text { nombre de sièges à pourvoir }: n=7
\end{array}\right.
$$

Les résultats de scrutin sont donnés dans le tableau I:

TABLEAU I

\begin{tabular}{|c|c|c|c|}
\hline & $x_{i}$ & $p_{i}$ & $n p_{i}$ \\
\hline Liste $1 \ldots \ldots \ldots$ & 420 & 0,420 & 2,940 \\
Liste $2 \ldots \ldots \ldots$ & 352 & 0,352 & 2,464 \\
Liste $3 \ldots \ldots \ldots$ & 228 & 0,228 & 1,596 \\
\hline
\end{tabular}

Méthode des plus forts restes: on commence par arrondir les proportions $n p_{i}$ de sièges à l'entier immédiatement inférieur et à réaliser ainsi une première affectation des sièges, ce qui donne:

$\left.\begin{array}{l}\text { liste } 1: 2 \text { sièges (reste } 0,94), \\ \text { liste } 2: 2 \text { sièges (reste } 0,464), \\ \text { liste } 3: 1 \text { siège (reste } 0,596 \text { ). }\end{array}\right\}$

Il reste deux sièges à pourvoir que l'on affecte par ordre décroissant des restes, ce qui donne la répartition définitive:

$$
\left.\begin{array}{l}
\text { liste } 1: 3 \text { sièges, } \\
\text { liste } 2: 2 \text { sièges, } \\
\text { liste } 3: 2 \text { sièges. }
\end{array}\right\}
$$


Méthode de la plus forte moyenne: on procède comme ci-dessus pour obtenir la première allocation de sièges (5). Le tableau II présente le déroulement de la méthode. On calcule pour les 3 listes la moyenne de suffrages pour un siège supplémentaire soit, par exemple pour la liste $1: 420 /(2+1)=140$. On ajoute un siège à la liste correspondant à la plus forte moyenne. On continue ainsi jusqu'à ce que tous les sièges soient pourvus.

TABLEAU II

\begin{tabular}{|c|c|c|c|c|c|}
\hline & \multicolumn{3}{|c|}{$\begin{array}{c}\text { Répartition } \\
\text { des } \\
\text { sièges }\end{array}$} & \multicolumn{2}{c|}{$\begin{array}{c}\text { Moyenne de suffrages } \\
\text { par } \\
\text { siège supplémentaire }\end{array}$} \\
\cline { 2 - 6 } & 1 & 2 & 3 & 1 & 2 \\
\hline Liste $1 \ldots \ldots \ldots$ & 2 & 3 & 3 & $140^{*}$ & 105 \\
Liste $2 \ldots \ldots \ldots$ & 2 & 2 & 3 & 117,3 & $117,3^{*}$ \\
Liste $3 \ldots \ldots \ldots$ & 1 & 1 & 1 & 114 & 114 \\
\hline
\end{tabular}

Sur cet exemple, on constate bien que la répartition à la plus forte moyenne défavorise la liste la plus faible, c'est-à-dire la liste 3 .

\subsection{Commentaires}

Les modes de calcul des deux méthodes semblent inspirés de bons sentiments: la méthode PFR postule qu'une liste est d'autant plus lésée que son reste est important; la méthode PFM considère qu'un siège non pourvu doit revenir à celui qui le mérite le plus a posteriori du point de vue du nombre de suffrages par siège. Où se trouvent l'équité et la justice dans tout cela? Il est bien difficile de répondre si l'on s'en tient uniquement à l'examen des modes de calcul qui sont en fait la transcription opératoire de certains objectifs sous-jacents. En effet, nous allons montrer comment chacune de ces méthodes revient à résoudre un problème de minimisation en nombres entiers de la forme:

$$
\left.\begin{array}{c}
\underset{n_{i}}{\operatorname{Min}} V=\sum_{i=1}^{q} f_{i}\left(p_{i}, n_{i}\right), \\
\sum_{i=1}^{q} n_{i}=n, \\
n_{i} \in \mathbb{N} .
\end{array}\right\}
$$

Chaque méthode correspondant à un critère $V$ différent et chaque mode de calcul présenté étant un algorithme particulier de résolution du problème 
d'optimisation associé. Nous étudierons successivement la méthode PFR et la méthode PFM.

\section{PROBLÈME D'OPTIMISATION ASSOCIÉ A LA METHODE DES PLUS FORTS RES- TES}

Nous établirons tout d'abord une propriété qui caractérise la répartition des sièges obtenus par la méthode PFR, nous montrerons ensuite qu'elle constitue la condition d'optimalité d'un problème de type (7)-(9) dont le critère $V$ est la dispersion quadratique moyenne entre la distribution des sièges et celle des suffrages, soit:

$$
V_{1}=\frac{1}{q} \sum_{i=1}^{q}\left(p_{i}-\frac{n_{i}}{n}\right)^{2}
$$

\subsection{Propriété fondamentale de la répartition des sièges}

En appliquant le mode de calcul de la méthode PFR, on aboutit à une répartition des sièges $\left\{n_{i}\right\}$ telle que $\sum_{i=1}^{q} n_{i}=n$. La proposition suivante permet de caractériser cette répartition:

Proposition 1: La répartition de sièges $\left\{n_{i}\right\}$ obtenue par la méthode PFR vérifie les relations suivantes:

$$
\left[\left(n_{i}-n p_{i}\right)-\left(n_{j}-n p_{j}\right)+1\right] \geqq 0, \quad \forall i, j .
$$

Démonstration: compte tenu du mode de calcul de la méthode PFR qui, en définitive, consiste à arrondir $n p_{i}$ à l'entier immédiatement inférieur ou supérieur, il est clair que l'on a l'inégalité:

$$
\left|n_{i}-n p_{i}\right|<1, \quad \forall i
$$

La relation (11) est trivialement vraie pour $i=j$. Supposons donc que cette relation ne soit pas satisfaite pour $i$ et $j$ tels que $i \neq j$; ceci signifie simplement que :

$$
\mid\left(n_{i}-n p_{i} \mid-\left(n_{j}-n p_{j} \mid>1\right. \text {. }\right.
$$

Des relations (12) et (13), on déduit que $\left(n_{i}-n p_{i}\right)$ et $\left(n_{j}-n p_{j}\right)$ sont de signe contraire, soit par exemple $\left(n_{i}-n p_{i}\right)>0$ et $\left(n_{j}-n p_{j}\right)<0$. Si $\left(n_{i}-n p_{i}\right)$ est positif, cela signifie:

- qu'on a ajouté un siège à la liste $i$ à partir de la répartition initiale des parties entières de $n p_{i}$;

vol. $20, \mathrm{n}^{\circ} 2$, mai 1986 
- que son reste $\left[n p_{i}-\left(n_{i}-1\right)\right]$ était alors supérieur au reste $\left(n p_{j}-n_{j}\right)$ de la liste $j$ soit $\left[n p_{i}-\left(n_{i}-1\right)\right] \geqq\left(n p_{j}-n_{j}\right)$, ce qui établit la relation (11).

\section{2. Programme d'optimisation associé}

Considérons le programme d'optimisation suivant:

$$
\left.\begin{array}{c}
\operatorname{Min} V_{1}=\frac{1}{q} \sum_{i=1}^{q}\left(p_{i}-\frac{n_{i}}{n}\right)^{2}, \\
\sum_{i=1}^{q} n_{i}=n, \\
n_{i} \in \mathbb{N} .
\end{array}\right\}
$$

Le programme consiste à rechercher la répartition des sièges qui minimise la dispersion quadratique moyenne $V_{1}$ entre la distribution des sièges $\left\{n_{i} / n\right\}$ et celle des suffrages $\left\{p_{i}\right\}$. Si l'on tient pas compte de la contrainte d'intégralité (16), la solution est évidemment $n_{i}=n p_{i}$. Pour résoudre ce programme quadratique en nombres entiers, on pourrait appliquer des algorithmes généraux de type Branch and Bound (cf. Minoux, [2]); toutefois la structure du problème est d'une telle simplicité que l'on peut caractériser la solution optimale par un raisonnement direct. C'est le sens de la proposition suivante:

Proposition 2: Pour qu'une répartition de sièges $n_{i}$ soit solution optimale du programme (14)-(16), il faut que:

$$
\left[\left(n_{i}-n p_{i}\right)-\left(n_{j}-n p_{j}\right)\right]+1 \geqq 0, \quad \forall i, j .
$$

Démonstration: considérons une répartition de sièges $\left\{n_{i}\right\}$ réalisable, c'està-dire complète $\left(\sum n_{i}=n\right)$. Évaluons la variation $u_{i j}$ de la fonction objectif due au transfert d'un siège de la liste $j$ à la liste $i$ (avec $n_{j} \geqq 1$ et $i \neq j$ ):

$$
\left.u_{i j}=\frac{1}{n^{2} q}\left[\left(\left(n_{i}+1\right)-n p_{i}\right)^{2}-\left(n_{i}-n p_{i}\right)^{2}\right)+\left(\left(n_{j}-1-n p_{j}\right)^{2}-\left(n_{j}-n p_{j}\right)^{2}\right)\right] .
$$

Soit, tous calculs faits:

$$
u_{i j}=\frac{2}{n^{2} q}\left[\left(\left(n_{i}-n p_{i}\right)-\left(n_{j}-n p_{j}\right)+1\right]\right.
$$

Une condition nécessaire d'optimalité de ce problème de minimisation s'écrit donc:

$$
u_{i j} \geqq 0, \quad \forall i, j \mid n_{j} \geqq 1 .
$$

R.A.I.R.O. Recherche opérationnelle/Operations Research 
Pour obtenir la relation (17), il reste à vérifier que la relation (19) est encore vérifiée par $n_{j}=0$; la démonstration de ce point technique est donnée dans l'Annexe 1.

La proposition 2 donne une condition nécessaire d'optimalité; elle constitue également une condition suffisante (cf. annexe 2). Nous pouvons donc énoncer le théorème suivant qui découle immédiatement des résultats obtenus:

THÉoRÈme 1: La répartition de sièges obtenue par la méthode PFR correspond au minimum de la dispersion quadratique moyenne entre la distribution des suffrages et celle des sièges.

Ajoutons que la dispersion quadratique moyenne $V_{1}$ mesure la "fidélité» de la représentation parlementaire par rapport aux suffrages obtenus par les différentes listes. Ce critère est indépendant du nombre de listes, puisqu'il s'agit d'une moyenne. Il constitue ainsi un indicateur qui permet d'établir des comparaisons d'un département à l'autre.

\section{PROBLÈMe d'OPTIMISATION ASSOCiÉ A LA MÉThOde de LA PLUS FORTE MOYENNE}

De la même façon que précédemment, nous commencerons par établir une propriété fondamentale qui caractérise la répartition des sièges obtenue par la méthode PFM. Nous montrerons qu'elle constitue la condition d'optimalité du problème d'optimisation dont le critère est :

$$
V_{2}=\sum_{i=1}^{q} p_{i}\left(\sum_{k=1}^{n_{i}} \frac{1}{k}\right),
$$

qui est une approximation du critère:

$$
V_{2}^{\prime}=\sum_{i=1}^{q} p_{i} \log n_{i},
$$

dont l'interprétation s'avère plus intéressante.

\subsection{Propriété fondamentale}

La proposition suivante est l'analogue de la Proposition 1:

PRoposition3: La répartition de sièges $\left\{\bar{n}_{i}\right\}$ obtenue par la méthode PFM vérifie les relations suivantes:

$$
\mathrm{p}_{i} / \bar{n}_{i} \geqq p_{j} /\left(\bar{n}_{j}+1\right), \quad \forall i, j .
$$

vol. $20, \mathrm{n}^{\circ} 2$, mai 1986 
Démonstration: Deux cas doivent être distingués:

- $\left(\overline{n_{i}}-n p_{i}\right)>0$. Cela signifie que, au cours du déroulement de la méthode PFM, un ou plusieurs sièges supplémentaires ont été attribués à la liste $i$. Soit $t$ la dernière étape où une telle attribution a eu lieu et soit $\left\{n_{j}^{t}\right\}$ la répartition (incomplète) réalisée au terme de cette étape. Il est clair que :

$$
\bar{n}_{i}=n_{i}^{t}=n_{i}^{t-1}+1 ; \quad \bar{n}_{j} \geqq n_{j}^{t}=n_{j}^{t-1} .
$$

Si la liste $i$ a ainsi été choisie à l'étape $t$, c'est que:

$$
x_{i} /\left(n_{i}^{t-1}+1\right) \geqq x_{j} /\left(n_{j}^{t-1}+1\right),
$$

Soit à un facteur $1 / n$ près:

$$
p_{i} /\left(n_{i}^{t-1}+1\right) \geqq p_{j} /\left(n_{j}^{t-1}+1\right) .
$$

D'où, grâce aux relations (23) :

$$
p_{i} / \bar{n}_{i} \geqq p_{j} /\left(\bar{n}_{j}+1\right) .
$$

- $\left(\bar{n}_{i}-n p_{i}\right) \leqq 0$, soit $p_{i} / \bar{n}_{i} \geqq 1 / n$. D'autre part, il est clair que $\left(\bar{n}_{j}-n p_{j}+1\right)>0$, $\forall j$ puisque $\bar{n}_{j}$ est supérieur ou égal à la partie entière de $n p_{j}$; il en résulte que :

$$
p_{i} / \bar{n}_{i} \geqq 1 / n>p_{j} /\left(\bar{n}_{j}+1\right),
$$

ce qui achève la démonstration.

\section{2. Programmes d'optimisation associés}

Considérons le programme d'optimisation suivant:

$$
\left.\begin{array}{c}
\operatorname{Max} V_{2}=\sum_{i=1}^{q} p_{i}\left(\sum_{k=1}^{n_{i}} 1 / k\right), \\
\sum_{i=1}^{n} n_{i}=n, \\
n_{i} \in \mathbb{N} .
\end{array}\right\}
$$

Il s'agit d'un programme d'optimisation dont le critère n'est défini que pour des valeurs entières de $n_{i}$. Il est toutefois assez facile de montrer que la relation (22) en est la condition d'optimalité:

Proposition4: Pour qu'une répartition de sièges $\left\{\bar{n}_{i}\right\}$ soit solution optimale du programme (28), il faut et il suffit que:

$$
p_{i} / \bar{n}_{i} \geqq p_{j} /\left(\bar{n}_{j}+1\right), \quad \forall i, j .
$$

R.A.I.R.O. Recherche opérationnelle/Operations Research 
La démonstration est donnée dans l'Annexe 3.

La signification exacte du critère $V_{2}$ s'éclaire si l'on remarque qu'il constitue une valeur approchée, pour $n$ assez grand, de:

$$
V_{2}^{\prime}=\sum_{i=1}^{q} p_{i} \log n_{i}
$$

On définit ainsi le programme approché :

$$
\left.\begin{array}{c}
\operatorname{Max} V_{2}^{\prime}=\sum_{i=1}^{q} p_{i} \log n_{i}, \\
\sum n_{i}=n, \\
n_{i} \in \mathbb{N} .
\end{array}\right\}
$$

La solution optimale de ce programme est caractérisée par la proposition suivante :

Proposition5: Pour qu'une répartition de sièges $\left\{\tilde{n}_{i}\right\}$ soit solution optimale du programme (31) il faut que:

$$
p_{i} \log \left(1+\frac{1}{\tilde{n}_{i}}\right)+p_{j} \log \left(1-\frac{1}{\tilde{n}_{i}}\right) \leqq 0, \quad \forall i, j / n_{j} \geqq 1,
$$

démonstration analogue à celle de la Proposition 2 (pour la condition nécessaire).

Nous verrons dans le paragraphe suivant comment les solutions des programmes (28) et (31) coïncident, sauf peut-être pour de faibles valeurs de $n$.

\subsection{Méthode PFM et théorie de l'Information}

Si l'on ne tient pas compte des conditions d'intégralité sur les $n_{i}$, le critère $V_{2}^{\prime}$ atteint son maximum pour $n_{i}=n p_{i}$. Pour la commodité de l'interprétation, il est préférable de considérer le critère $V_{2}^{\prime \prime}$ dont la minimisation est équivalente à la maximisation du critère $V_{2}^{\prime}$ :

$$
V_{2}^{\prime \prime}=\sum_{i=1}^{p} p_{i} \log _{2}\left(\frac{n p_{i}}{n_{i}}\right)
$$

$V_{2}^{\prime \prime}$ est toujours positif et constitue une mesure de dispersion particulière autour du point $\left\{n p_{i}\right\}$, qui s'interprète aisément grâce à la théorie de l'information ( $c f$. Benzecri [1] p. 216) : le critère $V_{2}^{\prime \prime}$ n'est autre en effet que l'information mutuelle entre la distribution des sièges et celle des suffrages, cette dernière vol. $20, \mathrm{n}^{\circ} .2$, mai 1986 
étant la distribution de référence. Dès lors, on peut énoncer le théorème suivant :

THÉORÈME 2: La répartition obtenue par la méthode PFM correspond, lorsque $n$ n'est pas trop petit,' à l'information mutuelle minimum entre la distribution des sièges et celle des suffrages.

\section{COMPARAISONS ET COMMENTAIRES}

Nous avons ainsi montré comment chacune des deux méthodes pouvait s'interpréter comme la recherche de la répartition des sièges minimisant un certain critère de dispersion autour du point $\left\{n p_{i}\right\}$. La différence des résultats obtenus d'une méthode à l'autre tient donc à ce qui distingue les critères à minimiser. A cet égard, les critères $V_{1}$ et $V_{2}^{\prime \prime}$ présentent une différence fondamentale: le critère $V_{1}$ est une fonction symétrique des distributions $\left\{p_{i}\right\}$ et $\left\{n_{i} / n\right\}$ tandis que le critère $V_{2}^{\prime \prime}$ ne l'est pas. C'est précisément ce qui explique pourquoi la méthode PFM a tendance à avantager les listes les mieux placées en étant plus généreuse à leur endroit dans la répartition des restes. Un tel phénomène apparaît clairement si l'on se livre à une étude graphique du cas où deux listes sont en présence; cette analyse nous permettra également de voir en quoi les problèmes (28) et (31), versions exacte et approchée de la PFM, ont en pratique la même solution.

\section{1. Analyse graphique d'un scrutin à deux listes}

La figure 1 représente les résultats obtenus par la méthode PFR, la méthode PFM (versions exacte et approchée), dans le plan $\left\{n_{1}, n_{2}\right\}$.

- La solution fractionnaire $\left(n_{1}=n p_{1}, n_{2}=n p_{2}\right)$ se trouve à l'intersection de la droite $\mathrm{O} z$ d'équation $n_{2}=\left(p_{2} / p_{1}\right) n_{1}$ et de la droite $I_{1} I_{2}$ d'équation $\left(n_{1}+n_{2}\right)=n($ point $Z)$.

- La condition d'optimalité de la méthode PFR s'écrit ici:

$$
\begin{gathered}
\left(n_{1}-n p_{1}\right)\left(n_{2}-n p_{2}\right)+1 \geqq 0, \\
\left(n_{2}-n p_{2}\right)-\left(n_{1}-n p_{1}\right)+1 \geqq 0,
\end{gathered}
$$

soit en tenant compte des égalités $p_{1}+p_{2}=1$ et $n_{1}+n_{2}=n$ :

$$
n_{1} p_{2}-n_{2} p_{1}+1 / 2 \geqq 0 \quad \text { et } \quad n_{2} p_{1}-n_{1} p_{2}+1 / 2 \geqq 0,
$$

ce qui correspond à la zone comprise entre les deux droites $C_{1} c_{1}$ et $C_{2} c_{2}$ symétriquement situées autour de la droite $\mathrm{O} z$.

R.A.I.R.O. Recherche opérationnelle/Operations Research 


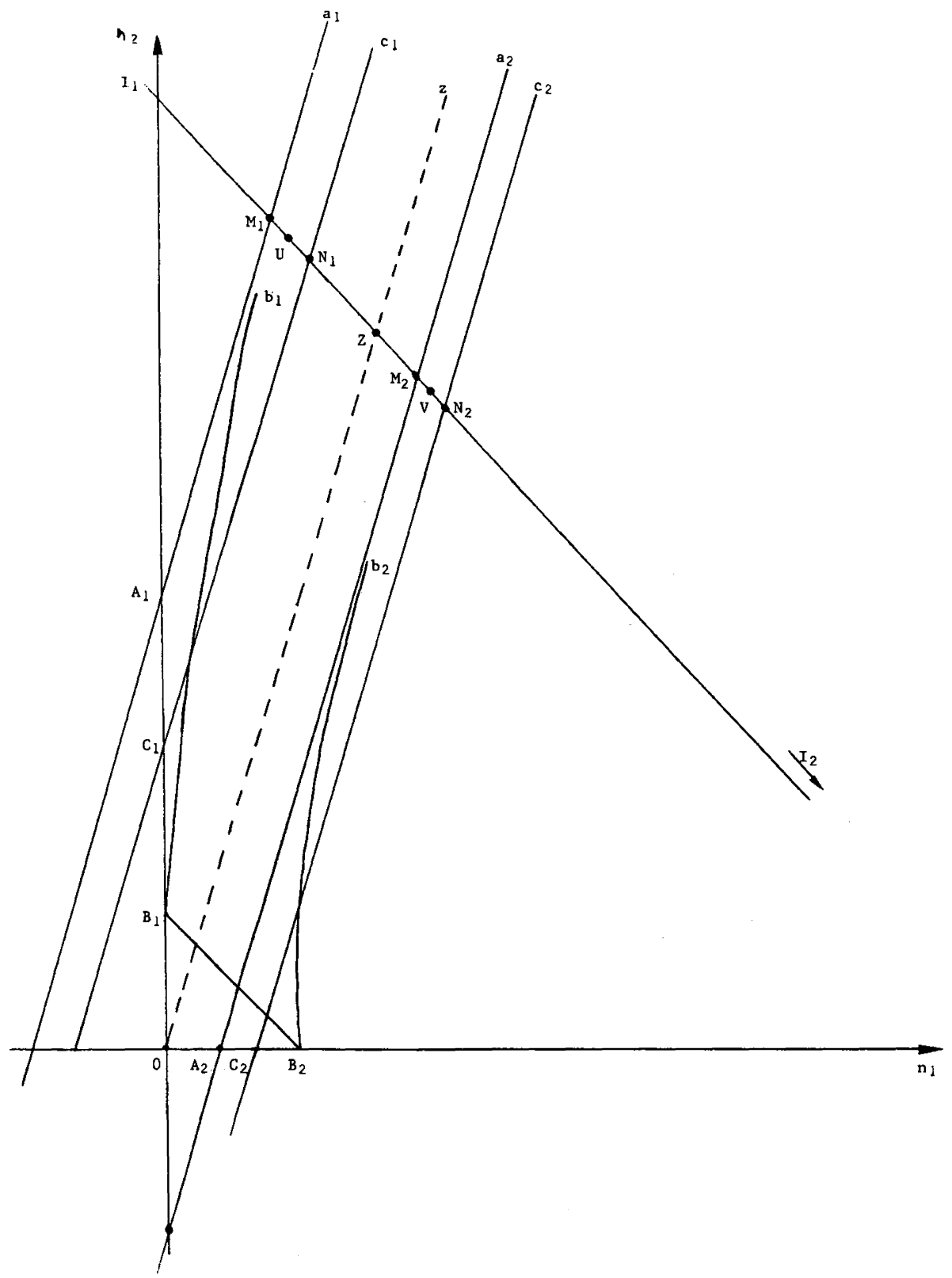

Figure 1. - Cas de deux listes $\left[n=7, p_{1}=3 / 13, p_{2}=10 / 13\right]$.

vol. $20, \mathrm{n}^{\circ} 2$, mai 1986 
- De manière similaire, la condition d'optimalité de la méthode PFM, version exacte, est satisfaite pour $\left\{n_{1}, n_{2}\right\}$ appartenant à la zone comprise entre les droites $A_{1} a_{1}, A_{2} a_{2}$ respectivement d'équations

$$
\left[n_{2} p_{1}-\left(n_{1}+1\right) p_{2}\right]=0 \text { et }\left[n_{1} p_{2}-\left(n_{2}+1\right) p_{1}\right]=0 \text {, }
$$

situées de manière non symétrique de part et d'autre de la droite $\mathrm{O} z$. Même raisonnement pour la version approchée avec les courbes $B_{1} b_{1}$ et $B_{2} b_{2}$ d'équations:

$$
\begin{gathered}
p_{1} \log \left(1+\frac{1}{n_{1}}\right)+p_{2} \log \left(1-\frac{1}{n_{2}}\right)=0, \\
p_{2} \log \left(1+\frac{1}{n_{2}}\right)+p_{1} \log \left(1-\frac{1}{n_{1}}\right) 0 .
\end{gathered}
$$

A noter que les courbes $B_{1} b_{1}$ et $B_{2} b_{2}$ tendent asymptotiquement vers les droites $A_{1} a_{1}$ et $A_{2} a_{2}$; ainsi les versions exactes et approchées fournissent la même solution sauf pour de faibles valeurs de $n$.

- La situation respective des droites $\left(A_{1} a_{1}, A_{2} a_{2}\right)$ et $\left(C_{1} c_{1}, C_{2} c_{2}\right)$ montrent bien comment la méthode PFM a tendance à privilégier des solutions entières placées au-dessus de la droite $\mathrm{O} z$ alors que la méthode PFR respecte une certaine symétrie autour de cette droite; cela signifie que la liste 2 sera plus avantagée que la liste 1 dans le cas de figure retenu ici $\left(p_{2} \gg p_{1}\right)$.

- Ceci apparaît clairement dans l'exemple numérique de la figure $1(n=7$, $\left.p_{1}=3 / 13, p_{2}=10 / 13\right)$ : la solution de la méthode PFR correspond au point $V\left(n_{1}=2, n_{2}=5\right)$ tandis que celle de la méthode PFM correspond au point $U$ $\left(n_{1}=1, n_{2}=6\right)$.

\section{2. Prolongements}

L'approche adoptée dans cet article présente un double avantage:

- Elle explicite les critères de choix réels qui déterminent la répartition des restes dans les deux méthodes. Fondamentalement ces deux méthodes se distinguent par des indicateurs de dispersion différents. La méthode, PFR s'inscrit dans une perception linéaire-quadratique des données, c'est-à-dire additive, tandis que la méthode PFM, qui s'appuie sur la Théorie de l'Information, est fondée sur une perception multiplicative. On retrouve là un clivage bien classique en statistique.

- Elle permet d'envisager d'autres critères débouchant sur d'autres modes de scrutin proportionnel, par exemple un critère quadratique de type $V_{1}$ pondéré par les proportions $p_{i}$ ou un critère du type:

$$
\sum_{i}\left(p_{i}-\frac{n_{i}}{n}\right) \log \left(\frac{n p_{i}}{n_{i}}\right)
$$

R.A.I.R.O. Recherche opérationnelle/Operations Research 
qui est symétrique et dont l'approximation du premier ordre n'est autre que le critère $V_{1}$. On peut également songer à des extensions, à des situations bidimensionnelles où il faut prendre en compte par exemple une répartition géographique et socio-professionnelle.

\section{CONCLUSION}

Les deux méthodes de répartition des restes que nous avons analysées présentent donc une structure commune: elles ne sont rien d'autre que des procédures itératives de résolution de deux problèmes d'optimisation. Ainsi, sait-on ce que l'on optimise: le débat peut s'engager sur l'essentiel: les critères de choix et non plus sur des résultats obtenus par des règles de calcul qui, pour l'électeur et l'élu moyens, demeurent assez mystérieuses.

\section{BIBLIOGR APHIE}

1. J. P. Benzecri, L'analyse des données, t. 1, Dunod, Paris, 1973.

2. M. Minoux, Programmation mathématique, Dunod, Paris, 1983.

3. B. Owen, Aux origines de lidée proportionnaliste, Pouvoirs, $\mathrm{n}^{\circ} 32,1985$, p. 15 à 38.

4. H. Portelli, Chronique politique, Projet, juillet-août, 1985.

5. J. Thе́рот et G. LeChenAult, Application de la classification hiérarchique à la coloration des graphes, R.A.I.R.O., vol. 15, $\mathrm{n}^{\circ} 1$, 1981, p. 73-83.

\section{ANNEXE 1}

Il faut montrer que la relation:

$$
\left[\left(n_{i}-n p_{i}\right)-\left(n_{j}-n p_{j}\right)+1\right] \geqq 0, \quad \forall i, j \mid n_{j} \geqq 1
$$

est encore vraie pour $n_{j}=0$.

Démontrons pour cela le lemme suivant:

LeMME: Soit $\left\{n_{i}\right\}$ une répartition de sièges vérifiant la condition $(A .1)$ alors:

(a) $\left(n_{i}-n p_{i}\right) \geqq-1$,

(b) $\left(n_{i}-n p_{i}\right) \leqq 1$.

Démontrons la relation $(a)$ : soit $K$ l'ensemble des listes ayant au moins un siège dans la répartition et soit $k=$ card $K$. En sommant la relation (A.1) pour $i$ fixé, l'on obtient:

$$
k\left(n_{i}-n p_{i}\right) \geqq-k+\sum_{j \in \mathbf{K}}\left(n_{j}-n p_{j}\right),
$$

vol. $20, \mathrm{n}^{\circ} 2$, mai 1986 
or $\sum_{j \in K} n_{j}=n$, d'où :

$$
k\left(n_{i}-n p_{i}\right) \geqq-k+n\left(1-\sum_{j \in k} p_{j}\right) \geqq-k,
$$

d'où la relation $(a)$. La relation $(b)$ s'obtient de manière analogue en sommant la relation (A.1) pour $j$ fixé et $n_{j} \geqq 1$ et en remarquant qu'elle est trivialement vraie pour $n_{j}=0$.

Pour $n_{j}=0$, le premier membre de la relation (A.1) s'écrit :

$\left(n_{i}-n p_{i}\right)+n p_{j}+1$ qui est positif grâce à la relation $(a)$ du lemme.

\section{ANNEXE 2}

\section{Condition suffisante d'optimalité}

La démonstration de la Proposition 2 est fondée sur un argument d'optimalité locale exprimant que l'on ne peut diminuer le critère $V_{1}$ par des transferts d'un siège d'une liste à une autre. Pour obtenir une condition suffisante, il faut établir que la relation (17) est une propriété d'optimalité globale: soient donc $\left\{n_{k}\right\}$ et $\left\{\tilde{n}_{k}\right\}$ deux distributions de sièges vérifiant la relation (17); nous allons montrer qu'elles correspondent à des valeurs égales du critère.

Grâce au lemme de l'Annexe 1, nous avons:

pour tout $i$ :

$$
\left|n_{i}-n p_{i}\right| \leqq 1, \quad\left|\tilde{n}_{i}-n p_{i}\right| \leqq 1, \quad \forall i
$$

Il en résulte donc que $\left|n_{i}-\tilde{n}_{i}\right| \leqq 2 ; n_{i}$ et $\tilde{n}_{i}$ étant des entiers, trois situations sont à considérer: $n_{i}=\tilde{n}_{i} \pm 2, \tilde{n}_{i}=n_{i} \pm 1$ et $n_{i}=\tilde{n}_{i}$. Éliminons d'emblée la première :

- Si il existe $i$ tel que $n_{i}=\tilde{n}_{i}+2$ (par exemple), alors, de la relation A. 3 il résulte que $n_{i} \leqq n p_{i}+1$ et $\tilde{n}_{i} \geqq n p_{i}-1$, soit $n_{i} \geqq n p_{i}+1$, c'est-à-dire $n_{i}=n p_{i}+1$. On déduirait alors de (17) que $n_{k}-n p_{k} \geqq 0$, pour tout $k \neq i$, d'où, en sommant, $n=n+1$; contradiction.

- Définissons donc $A=\left\{k . / . n_{k}=\tilde{n}_{k}\right\}, \quad B=\left\{k . / . \tilde{n}_{k}=n_{k}+1\right\} \quad$ et $C=\left\{k \cdot / \cdot n_{k}=\tilde{n}_{k}-1\right\}$. Soit $v$ et $\tilde{v}$ les valeurs du critère pris par les distributions $\left\{n_{k}\right\}$ et $\left\{\tilde{n}_{k}\right\}$. Si les ensembles $B$ et $C$ sont vides, les distributions sont identiques. Supposons donc (par exemple) $B \neq \varnothing$, il en résulte que 
$C \neq \varnothing$ puisque $\sum_{k} n_{i}=\sum_{k} \tilde{n}_{k}=n$. Autrement dit, s'il existe $i$ tel que $n_{i}=\tilde{n}_{i}+1$, il existe $j$ tel que $n_{j}=\tilde{n}_{j}-1$. La relation (17) donne donc:

$$
\begin{gathered}
\left(n_{j}-n p_{j}\right)-\left(n_{i}-n p_{i}\right)+1 \geqq 0, \\
\left(n_{i}-n p_{i}\right)-\left(\tilde{n}_{j}-n p_{j}\right)+1 \geqq 0 ;
\end{gathered}
$$

soit :

$$
\left(n_{j}-n p_{j}\right)-\left(\tilde{n}_{i}-n p_{i}\right) \geqq 0
$$

et

$$
\left(\tilde{n}_{i}-n p_{i}\right)-\left(n_{j}-n p_{j}\right) \geqq 0 .
$$

Il en résulte alors que:

$$
\left(n_{j}-n p_{j}\right)=\left(\tilde{n}_{i}-n p_{i}\right) \quad \text { et } \quad\left(n_{i}-n p_{i}\right)=\left(\tilde{n}_{j}-n p_{j}\right) .
$$

En clair, cela signifie qu'en remplaçant $n_{i}$ par $\tilde{n}_{i}$ et $n_{j}$ par $\tilde{n}_{j}$ dans la distribution $\left\{n_{k}\right\}$, on obtient une distribution $\left\{n_{k}^{\prime}\right\}$ dont la valeur du critère est toujours $v$. Il suffit alors de définir les ensembles $A^{\prime}, B^{\prime}, C^{\prime}$ à partir de la distribution $\left\{n_{k}^{\prime}\right\}$ en remarquant que card $B^{\prime}=\operatorname{card} B-1$ et card $C^{\prime}=C-1$. En répétant. l'opération autant de fois qu'il est possible on aboutit à des ensembles $B^{*}$ et $C^{*}$ vides et une distribution $\left\{n_{k}^{*}\right\}$ qui prend alors la valeur $\tilde{v}$. De proche en proche, on a montré ainsi que $v=\tilde{v}$.

\section{ANNEXE 3}

\section{Démonstration de la proposition 3}

\section{CONDITION NEECESSAIRE}

Comme dans la démonstration de la proposition 2, nous allons évaluer la variation $w_{i j}$ la fonction-objectif due au transfert d'un siège de la liste $i$ à la liste $j\left(\right.$ avec $\bar{n}_{i} \geqq 1$ et $\left.i \neq j\right)$ :

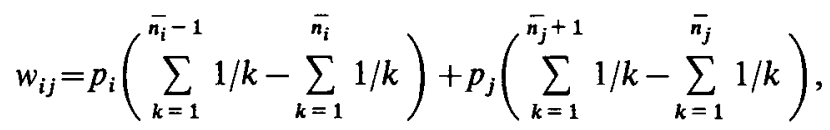

soit $w_{i j}=-p_{i} / \bar{n}_{i}+p_{j} /\left(\bar{n}_{j}+1\right)$. Une condition nécessaire d'optimalité s'écrit $w_{i j} \leqq 0$ d'où la relation (22).

vol. $20, n^{\circ} 2$, mai 1986 
La relation (22) est trivialement vérifiée pour $\bar{n}_{i}=0$. Si l'on admet la convention $k / 0=+\infty$.

\section{CONDITION SUFFISANTE}

Soient $\left\{n_{k}\right\}$ et $\left\{\tilde{n}_{k}\right\}$ deux distributions de sièges vérifiant la relation (22) correspondant à des valeurs $v$ et $\tilde{v}$ du critère $V_{2}$. Montrons que $v=\tilde{v}$. Définissons $A=\left\{k . / . n_{k}=\tilde{n}_{k}\right\}, B=\left\{k . / . n_{k}>\tilde{n}_{k}\right\}$ et $C=\left\{k . / . n_{k}<\tilde{n}_{k}\right\}$. Si $B \neq 0$ alors $C \neq 0$; soient donc $i \in B$ et $j \in C$. Nous avons alors $n_{i}=\tilde{n}_{i}+s$, $n_{j}=\tilde{n}_{j}-r$ avec $r$ et $s \geqq 1$. La relation (22) donne les deux inégalités:

$$
p_{i} / n_{i} \geqq p_{j} /\left(n_{j}+1\right), \quad p_{j} / \tilde{n}_{j} \geqq p_{i} /\left(\tilde{n}_{i}+1\right) ;
$$

Soit :

$$
p_{i} /\left(\tilde{n}_{i}+s\right) \geqq p_{j} /\left(n_{j}+1\right), \quad p_{j} /\left(n_{j}+r\right) \geqq p_{i} /\left(\tilde{n}_{i}+1\right),
$$

d'où les inégalités:

$$
p_{i} /\left(\tilde{n}_{i}+s\right) \geqq p_{j} /\left(n_{j}+1\right) \geqq p_{j} /\left(n_{j}+r\right) \geqq p_{i} /\left(\tilde{n}_{i}+1\right),
$$

qui ne peuvent être que des égalités avec $r=s=1$. En remplaçant $n_{i}$ par $\tilde{n}_{i}$ et $n_{j}$ par $\tilde{n}_{j}$ dans la distribution $\left\{n_{k}\right\}$ on obtient ainsi une distribution de sièges dont la valeur du critère reste égale à $v$. Le raisonnement de la fin de l'annexe 3 peut être appliqué alors pour aboutir à la conclusion. 